\title{
chemosensors
}

ISSN 2227-9040

www.mdpi.com/journal/chemosensors

Editorial

\section{Acknowledgement to Reviewers of Chemosensors in 2014}

Chemosensor Editorial Office, MDPI AG, Klybeckstrasse 64, CH-4057 Basel, Switzerland

Published: 13 January 2015

The editors of Chemosensors would like to express their sincere gratitude to the following reviewers for assessing manuscripts in 2014:

$\begin{array}{lll}\text { Algar, W. Russ } & \text { Jia, Ze } & \text { Shim, Yoon-Bo } \\ \text { Asher, Prof. Dr. Sanford } & \text { ju, huangxian } & \text { Su, Pi-guey } \\ \text { Benavente, J. } & \text { Lo, Yu-Lung } & \text { Sung, Ti-Wen } \\ \text { Buonsanti, Raffaella } & \text { Lorenzo, E. } & \text { Szmacinski, Henryk } \\ \text { Charles, Paul T. } & \text { Martucci, Alessandro } & \text { Szymanski, Wiktor } \\ \text { Chen, Jiajun } & \text { Matsumiya, Masahiko } & \text { Tang, Zhenghua } \\ \text { Galstyan, Vardan } & \text { Mendes, Eduardo } & \text { Tsai, Yu-Chen } \\ \text { González Monrroy, Javier } & \text { Nielsen, Poal } & \text { Vergara, Alexander } \\ \text { Gutiérrez-Capitán, Manuel } & \text { Park, Jungyul } & \text { Wencel, Dorota } \\ \text { Haghiri-Gosnet, A. M. } & \text { Pesavento, Maria } & \text { Wong, Danny K. Y. } \\ \text { Haick, Hossam } & \text { Rubis, Blazej } & \text { Yamaguchi, Akira } \\ \text { hHrdlicka, Patrick } & \text { Rudnitskaya, Alisa } & \text { Yu, Aimin } \\ \text { Huang, Bohr-ran } & \text { Shen, Wen } & \text { Yusof, Nor Azah }\end{array}$

(C) 2015 by the authors; licensee MDPI, Basel, Switzerland. This article is an open access article distributed under the terms and conditions of the Creative Commons Attribution license (http://creativecommons.org/licenses/by/4.0/). 